J Child Adolesc Psychopharmacol Vol. 4(3):171-182 (1994)

ISSN: (print 1044-5463)(online 1557-8992)

doi:10.1089/cap.1994.4.171

This is a peer reviewed pre-print version of the following article: Potential Link Between Dietary Intake of Fatty Acids and Behavior: Pilot Exploration of Serum Lipids in Attention-Deficit Hyperactivity Disorder, which has been published in final form at:

http://www.liebertpub.com

http://www.liebertpub.com/cap

http://www.liebertonline.com/doi/pdfplus/10.1089/cap.1994.4.171

(C) 1994 Mary Ann Liebert, Inc. Publishers

\title{
Potential Link Between Dietary Intake of Fatty Acids and Behavior: Pilot Exploration of Serum Lipids in Attention-Deficit Hyperactivity Disorder
}

\author{
L. Eugene Arnold, M.Ed., M.D, Professor Emeritus of Psychiatry, Ohio State \\ University, and Special Expert, Child and Adolescent Disorders Research Branch, \\ National Institute on Mental Health., Donald Kleykamp, M.D., Wright State \\ University, Dayton, Ohio., Nicholas Votolato, Pharm.D., Ohio State University, \\ Columbus, Ohio., Robert A. Gibson, Ph.D., Flinders Medical Centre, Adelaide, South \\ Australia., andLloyd Horrocks, Ph.D. Ohio State University, Columbus, Ohio.
}

\begin{abstract}
Essential fatty acids are structural components of all biological membranes and form the environment for membrane receptors, ion channels, and enzymes. Dietary linoleic acid is metabolized by delta-6-desaturase to gamma-Iinolenic acid (GLA). Through several further metabolic steps, GLA is eventually converted to series-1 prostaglandins, thromboxanes, and other molecules involved in regulating the moment-to-moment function of various physiological processes. For example, prostaglandins interact with dopamine neurotransmission. The biochemistry and physiology of essential fatty acids are reviewed, with attention to possible implications for behavior. Delta-6-desaturase deficiency has been hypothesized to be one cause of attention-deficit hyperactivity disorder (ADHD). To explore this possibility, we examined the correlations of serum lipids and behavior in a double-blind crossover comparison of GLA, d-amphetamine, and placebo in 16 boys (ages 6-12) with ADHD. Looking at the fatty-acid components of serum triglycerides across treatment conditions, we found that higher scores for behavior problems correlated with lower levels of GLA (for each of four behavioral ratings, $\mathrm{p}<0.015$ ) but not with its precursor, linoleic acid. This is compatible with a metabolic bottleneck at delta-6-desaturase being related to some ADHD symptoms. These preliminary findings are consistent with the possibility that fatty-acid intake or metabolism may influence behavior, at least in children with ADHD, and could conceivably modulate the effects of psychopharmacological treatment. This study must be considered exploratory and heuristic, and more definitive studies are needed to examine the possible relevance of fatty-acid metabolism to psychiatric disorders.
\end{abstract}

Several possible mechanisms may link essential fatty-acid metabolism to behavior. These potential connections include not only the effects of general body metabolism on brain function, but also the structural need for polyunsaturated fatty acids in neuronal membranes and the influence of these fatty acids and their metabolic products on neurotransmitter production, release, and effects.

This article will review the biochemistry and physiology of essential fatty acids, consider some suggestive animal behavior findings, focus more specifically on possible implications of gamma-linolenic acid (GLA) for attention-deficit hyperactivity disorder (ADHD), and finally present some relevant pilot data.

\section{Background Biochemistry of Essential Fatty Acids}

Fatty acids are long hydrogenated carbon chains with a carboxyl group (-COOH) at one 
end. Essential fatty acids are certain polyunsaturated fatty acids that have several double bonds between carbon atoms with the potential of being "saturated" with additional hydrogen atoms (see Fig. 1).

Fatty acids can have unsaturated intercarbon double-bonds at every third position from the tail (the end opposite the carboxyl group). Plants can enzymatically desaturate any of the double bonds, but animals can only desaturate at position 9 and above, counting from the tail. Since most animals require fatty acids that are unsaturated in the three and six positions and cannot make them, these "essential" fatty acids must be ingested.

The essential fatty acids fall into two groups: The omega-3 series $(\Omega 3$ or $n-3)$ and the omega-6 series (Q6 or n-6) are shown in Fig. 2. The "most essential" is the $\Omega 6$ series, which predominates over the $\Omega 3$ series by a ratio of $3: 1$ to $6: 1$ in most tissues except neural, where the ratio is about equal (Horrobin and Manku 1990).

Replenishment with $\Omega 6$ essential fatty acids alone seems to reverse most essential fatty-acid deficiency symptoms, but replenishment with $\Omega 3$ essential fatty acids seems ineffective in the face of continuing $\Omega 6$ deficiency, so there was controversy for awhile as to whether the $\Omega 3$ series was essential at all. However, more recent research has established subtle brain and retinal dysfunction resulting from $\Omega 3$ deficiency alone (Philbricketal. 1987, Neuringeretal. 1988, Bourreetal. 1989). Enslenetal. (1991) found in $\Omega 3$-deprived rat pups a reduced level of normal exploratory behavior associated with substitution of $\Omega 6$ for $\Omega 3$ essential fatty acids in brain phospholipids.

It is not necessary for most normal humans to ingest all acids in a series because human physiology can manufacture the rest of the series from the first in each series, by alternating steps of further desaturation and elongation.

Conventional designation of a specific fatty acid first states the number of carbon atoms (before the colon), then (immediately after the colon) the number of unsaturated bonds, and finally the position of the first unsaturated bond (counting from the tail). For example, 18:2 $\Omega 6$ (or 18:2n-6) is an 18-carbon acid with 2 unsaturated bonds at positions 6 and 9 from the tail. This compound, 18:2 $\Omega 6$, happens to be linoleic acid, the parent compound in the $\Omega 6$ series (see Fig. 1). Linoleic acid is abundant in most temperate vegetable oils and is even present
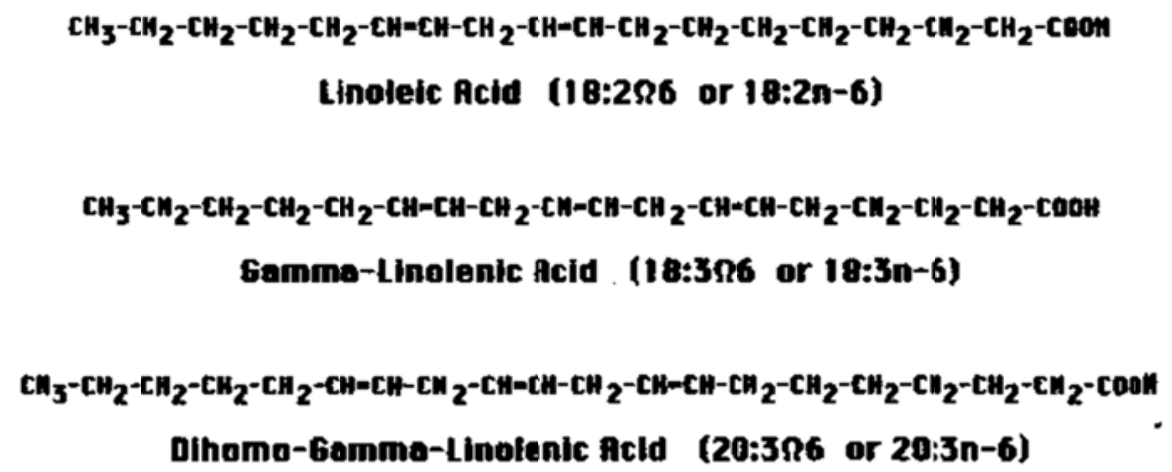

Fig. 1. Structure of fatty acids. Fatty acids are hydrogenated carbon chains with a carboxyl group (-COOH) on one end. The double bonds between the carbon atoms are "unsaturated," that is, not saturated with hydrogen atoms. Single bonds allow the carbon atom to have two hydrogen atoms. The end ("tail") carbon atom has 3 hydrogen atoms. The position of the double-bonds is counted from the tail (-CH3). 


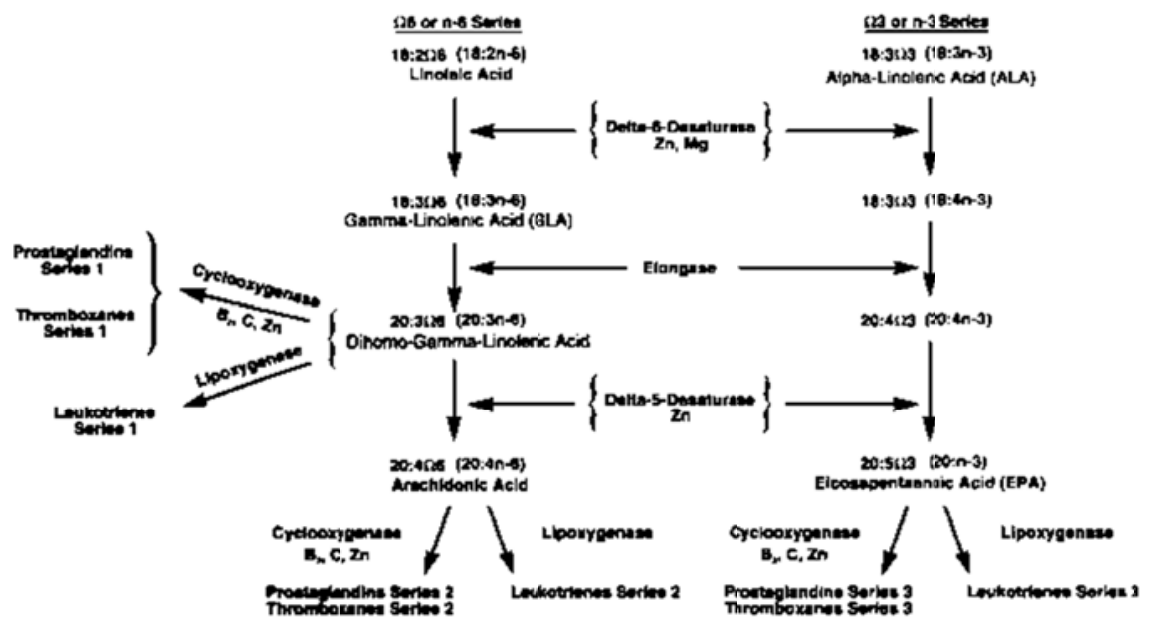

Fig. 2. The twin series of essential fatty acids and their regulatory metabolites: The $\Omega 6$ or n- 6 series has the first unsaturated bond at the sixth carbon from the tail (opposite end from carboxyl group) and provides the precursors for the series- 1 and series- 2 eicosanoids (20-carbon regulatory metabolites). The $\Omega 3$ or n- 3 series, providing the precursor for the series-3 eicosanoids, has the first unsaturated bond at the third carbon from the tail, and at each metabolic step has one more unsaturated bond than the homologous $\Omega 6$ essential fatty acid. Conventional notation of essential fatty acids is described in text.

in animal fat. Similarly, 18:3 $\Omega 3$, or 18:3n-3, is alpha-linolenic acid (not to be confused with gamma-linolenic acid, $18: 3 \Omega 6$ or 18:3n-6), which is the parent compound of the $\Omega 3$ series; it is also found in a wide range of diets.

Both series use the same desaturase and elongase enzymes, leading at times to competitive interference, especially of desaturation. The $\Omega 3$ series seems to compete more successfully for desaturase, thereby inhibiting desaturation of the $\Omega 6$ series (Horrobin and Manku 1990, Sprecher 1982).

The rate-limiting step, especially for synthesis of the $\Omega 6$ series, is the first enzyme, delta-6-desaturase. Enzymatic desaturation can be further impaired by aging, diabetes, high alcohol intake, high cholesterol, or liver disorder (Brenner 1982, Horrobin and Manku 1990). The alcohol inhibition of delta-6-desaturase has been suspected to be a mechanism for fetal alcohol effects (Varma 1982). In addition, there are interactions with mineral nutrition, since zinc and magnesium are coenzymes with delta-6-desaturase (e.g., Bettger et al. 1979).

\section{Regulatory metabolites}

Essential fatty acids with 20 carbons are converted by a variety of metabolic routes to eicosanoids (prostaglandins, thromboxanes, leukotrienes, lipoxins, hydroperoxy- and epoxy-acids); these 20-carbon ("eicosa") metabolites regulate most tissues, including neural (Horrobin and Manku 1990). In some of their actions they act much like neurotransmitters, or at least coagents. They act as second messengers and modulate synaptic transmission (Wolfe and Horrocks 1993).

Prostaglandins (PGs) and thromboxanes (TXs) are formed by cyclooxygenase, which is inhibited by anti-inflammatory agents, including aspirin (Gomez-Nino et al. 1992). They are distinguished by their ring structure ("cyclo") from other eicosanoids formed by other enzymes. The 1-series (e.g., $\mathrm{PGE}_{1}, \mathrm{PGF}_{1}$ ) derives from dihomo-gamma-linolenic acid (dihomo-GLA, DHGLA, 20:3 26 ). The 2-series (e.g., $\mathrm{PGE}_{2}, \mathrm{PGF}_{2}, \mathrm{TXA}_{2}$ ) is derived from arachidonic acid

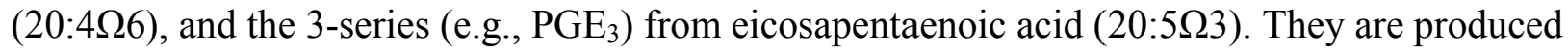


directly from the essential fatty-acid precursor in each tissue and quickly inactivated, thus regulating the moment-to-moment function of various physiological processes (Horrobin and Manku 1990). There are interactions with other essential nutrients: zinc, ascorbate (vitamin C), and niacin (B3) are coenzymes with cyclooxygenase, and series-2 prostaglandins are believed to be necessary for zinc absorption from the gut (Song and Adham 1980).

Other eicosanoids include the leukotrienes (formed by lipoxygenase) and the epoxy-acids (formed by cytochrome P-450, which is inhibited by some psychotropic drugs and induced by others).

\section{Essential Fatty-Acid Activity and Neurons}

The concept of essential fatty-acid activity includes both the effects of the fatty acids themselves and the effects of their metabolites, mainly eicosanoids. Essential fatty acids are essential constituents of all membranes from skin and gut to neuronal cell membranes, and affect plasticity and permeability. They form the environment for membrane proteins such as receptors, ion channels, and enzymes. A high proportion of unsaturation in a membrane reduces steroid binding to receptors in that membrane (Horrobin and Manku 1990, Mitsuhashi et al. 1986). Unsaturated fatty acids can block cell-membrane chloride channels (Hwang et al. 1990), activate $\mathrm{K}+$ channels (Kim and Clapham 1989, Ordway et al. 1989), and activate the $\mathrm{d}$ isoform of protein kinase C (McPhail et al. 1984). Crane and Greenwood (1987) found dietary fat to influence neuronal monoamine oxidase activity (high level of dietary polyunsaturates suppressed mitochondrial MAO).

Long-term potentiation of synaptic transmission in the hippocampus is a persistent enhancement of synaptic efficacy precipitated by brief, high frequency, tetanic glutamate-pathway activation (Bliss et al. 1990). It has been implicated in learning and memory. Glutamate depolarizes the postsynaptic membrane, relieving the $\mathrm{Mg}^{2+}$ blockade of the NMDA receptor ion channel and letting calcium enter to initiate the cascade that persistently enhances synaptic transmission. A current mechanism hypothesis is that arachidonic acid is released from postsynaptic membranes and diffuses to the presynaptic terminal, where it facilitates glutamate release (Wolfe and Horrocks 1993).

Prostaglandins (eicosanoid derivatives of essential fatty acids) have many interactions with dopamine and norepinephrine, the catecholamine neurotransmitters that have been implicated in ADHD and other psychiatric and behavioral disorders. For example, dopamine $\left(D_{2}\right)$ receptor stimulation potentiates release of arachidonic acid for $\mathrm{PGE}_{2}$ synthesis; high levels of $\mathrm{D}_{2}$ receptors and $\mathrm{PGE}_{2}$ in such CNS areas as hypothalamus and amygdala suggest a modulatory role for $\mathrm{PGE}_{2}$ in dopamine transmission (Di Marzo and Piomelli 1992). Prostaglandins and norepinephrine act antagonistically on Purkinje cell firing; $\mathrm{PGE}_{2}$ reduces neostriatal and cortical release of dopamine and norepinephrine; dopamine stimulates phospholipase-A mobilization of prostaglandin precursors from ester bonds; the binding of biogenic amines to postsynaptic receptors releases prostaglandins into the synaptic cleft, which inhibit neurotransmitter release through calcium-mediated negative feedback to the presynaptic neuron (Gross et al. 1977, Molderings et al. 1992, Gomez-Nino et al. 1992). Sympathetic (and parasympathetic) stimulation releases prostaglandins, probably synthesized in the postsynaptic effector cell de novo (Sherbourne et al. 1992); $\mathrm{PGE}_{2}$ may inhibit the presynaptic release of norepinephrine (Wolfe and Horrocks 1993, Racke et al. 1992). However, Negishi and Ito (1992) reported that $\mathrm{PGE}_{2}$ induced catecholamine secretion from cultured bovine adrenal chromaffin cells. Catecholamines, acting as cosubstrates, stimulate prostaglandin synthesis and release, but inhibit leukotriene synthesis (Alanko et al. 1992, Nadasy et al. 1992). Regulation of prostaglandin production in rat brain requires intact 
central adrenergic function (Weidenfeld et al. 1992). The MAO inhibitor tranylcypromine inhibits prostacyclin synthetase (Xiang and McNeil 1992). Central prostaglandins may provide the mechanism for catalepsy (Ono et al. 1992). In the hypothalamus, norepinephrine releases $\mathrm{PGE}_{2}$, mediated by nitric oxide (Rettori et al. 1992). The prostaglandins modulate pituitary hormone release by modulating dopamine (and other neurotransmitter) release; for example, $\mathrm{PGD}_{2}$ induces LH release (Wolfe and Horrocks 1993).

Prostaglandins, especially $\mathrm{PGE}_{1}$, also raise cyclic AMP and cyclic GMP activity postsynaptically (Horrobin and Manku 1990, Rettori et al. 1992), an effect inhibited by opioids (Wolfe and Horrocks 1993). Some animal studies also have suggested interaction of prostaglandins and serotonin, another neurotransmitter sometimes implicated in ADHD and other psychiatric disorders; for example, $\mathrm{PGD}_{2}$ increases brain serotonin content and turnover (Wolfe and Horrocks 1993). Further, $\mathrm{PGD}_{2}$ in the preoptic hypothalamus induces sleep (Hayaishi 1991) while $\mathrm{PGE}_{2}$ induces wakefulness.

In view of these biochemical findings, we might suspect that there may be some behavioral effects of dietary intake of the different lipids.

\section{Behavioral Effects in Animal Studies}

While most animal studies of dietary fat manipulation focus on biochemical, caloric, food choice, and body weight effects, there have been some provocative observations of learning and other behaviors. As compared to a 20\% lard (saturated fat) diet, an otherwise identical $20 \%$ soybean-oil (polyunsaturated) diet results in improved learning on a variety of tasks, increased pain tolerance, increased preference for carbohydrate over protein, and increased exploratory activity in rats (Yehuda and Carasso 1987, Yehuda et al. 1986, Coscina et al. 1986, Greenwood and Winocur 1990). The improved learning appeared within 3 weeks and was transiently resistant to extinction. It could not be explained by basal body temperature, activity level, energy consumption, body weight, or brain choline acetyltransferase levels (Coscina et al. 1986). The improved learning apparently depends on a balance between $\Omega 3$ and $\Omega 6$ essential fatty acids (as found in soybean oil), not just essential fatty acids in general: Bourre et al. (1989) found that a sunflower-oil diet (highly polyunsaturated but only $\Omega 6$; poor in $\Omega 6$ essential fatty acids) actually impaired shuttle-box learning.

Enslen et al. (1991) report an interesting developmental implication. Four-month-old rats whose mothers had been gestationally fed either a $6 \%$ soybean-oil (balanced $\Omega 3$ and $\Omega 6$ essential fatty acids) diet or an otherwise identical $5.5 \%$ safflower-oil (rich in $\Omega 6$, poor in $\Omega 3$ essential fatty acids) diet were compared behav-iorally and histochemically. The total polyunsaturated 22-carbon fatty acids in synaptic membrane and myelin phospholipids were the same for both-conditions, but the ratio of $22: 6 \Omega 3$ to $22: 5 \Omega 6$ was significantly decreased in the safflower-oil-gestated rat brains. The safflower-oil rats also showed significantly lower exploratory behavior in novel environments. Feeding soybean-oil diets from weaning on could correct the brain phospholipid 22:6 $\Omega 3$-to-22:5 $\Omega 6$ ratio, but could not normalize the reduced exploratory behavior, suggesting a specific developmental need.

\section{Gamma-Linolenic Acid and Attention-Deficit Hyperactivity Disorder}

Some children are hypothesized to be deficient in delta-6-desaturase, which converts dietary linoleic acid (C18:2Q6) to gamma-linolenic acid (GLA, C18:3Q6), the precursor of dihomo-gamma-linolenic acid (dihomo-GLA, DHGLA, C20:3Q6), the only precursor for prostaglandin series-1. Widespread infantile vulnerability of this enzyme in the human species is suggested by the relatively large amount of GLA in human milk (Gibson and Kneebone 1981) as 
compared to other species, such as goats and cows. The fact that it is not present in all species suggests a selective evolutionary value, with active secretion rather than leak from the blood. Thus, it is reasonable to suspect that many human infants have immature delta-6-desaturation (which is compensated for by the presence of GLA in mother's milk). Taylor and Wadsworth (1984), reporting a slight but statistically significant cognitive and behavioral advantage of breast-fed over bottle-fed infants, speculated that one explanation might be that"... particular constituents of human milk may provide particular nutrients which allow optimal development of the human brain ..." (p. 78). Some children may have later maturation than others, resulting in symptoms associated with prostaglandin series- 1 and possibly series- 2 delta-6-desaturase deficiency. Because of the interactions between prostaglandins and catecholamine transmission described above, such relative deficiency states might cause symptoms of disorders that involve catecholamine aberrations, such as ADHD (Colquhoun and Bunday 1981). Supplementation with GLA should bypass defective delta-6-desaturation and correct such hypothesized relative deficiencies.

Colquhoun and Bunday (1981) reported, in an open study, an apparent benefit of GLA in treating children with ADHD. We have reported (Arnold et al. 1989) a double-blind placebo-controlled crossover comparison of GLA supplementation to dextroamphetamine in 18 boys with ADHD. All 3 treatment conditions showed a significant difference from each other on the hyperactivity factor of the Conners 39-item teacher rating, and showed a similar but nonsignificant trend on other measures. Overall, these trends suggested a possible benefit of GLA use, but the effect appeared smaller than with dextroamphetamine treatment. Mitchell et al. (1987) reported significantly lower levels of serum dihomo-GLA (20:3Q6, precursor of series-1 eicosanoids), arachidonic acid (20:4 $\Omega 6$, precursor of series-2 eicosanoids), and docosahexaenoic acid (22:6 $\Omega 3)$ in 48 hyperactive children than in 49 age- and sex-matched controls. However, Aman et al. (1987) failed to find significant correlations of baseline blood lipids and behavior ratings in hyperactive children.

To the best of our knowledge, no investigators have previously examined the correlation of blood lipids and behavior during treatment in a double-blind placebo-controlled crossover study. If essential fatty-acid metabolism and GLA supplementation truly affect behavior, we should expect significant correlations (regardless of treatment conditions) between behavioral scores and measures of body essential fatty-acid status. More specifically, delta-6-desaturase is the rate-limiting step in the synthesis of GLA (Horrobin and Manku 1990, Sprecher 1982, Brenner 1982): If defective delta-6-desaturation of linoleic acid is an enzymatic bottleneck that is contributing to behavior problems, we would expect that (across conditions) behavior problems would correlate significantly with GLA (the product of delta-6-desaturation) but not with linoleic acid (the substrate). Behavior should also correlate significantly with the ratio of GLA (the downstream metabolite) to linoleic acid (the precursor). We report here a pilot exploration of this hypothesis.

\section{Method}

The subjects of this pilot exploration were a convenience sample in a previously reported (Arnold et al. 1989) double-blind completely counterbalanced double-crossover study of 18 ADDH boys age 6-12 (mean and median age 9) comparing one month each of placebo, dextroamphetamine, and GLA supplementation. All subjects had a diagnosis of ADHD by DSM-III criteria (determined clinically by a child psychiatrist), normal intelligence, a score of 18 or more on the Conners 10-item Hyperactivity Index, a sum of 24 or more on the first 6 items of the Davids Hyperkinetic Rating Scale (Davids 1971), no history of seizures, and no psychoactive 
drug in the preceding week.

The GLA was administered in the form of EffamolTM capsules, each containing $500 \mathrm{mg}$ evening primrose oil, including $350 \mathrm{mg}$ linoleic acid and $40 \mathrm{mg}$ GLA. The dose of Effamol ${ }^{\mathrm{TM}}$ was four capsules twice daily at breakfast and supper; it furnished a total daily dose of $320 \mathrm{mg}$ of GLA. It was assumed that, with the wide availability and variation of linoleic acid in the ordinary American diet, the additional 2.8 grams of linoleic acid would not in itself produce a significant behavioral effect; so its presence in the Effamol ${ }^{\mathrm{TM}}$ was presumed to be inert. The dose of dextroamphetamine (either a 10- or 15-mg time-released spansule administered at breakfast) was selected to provide between $0.35 \mathrm{mg}$ and $0.70 \mathrm{mg} / \mathrm{kg}$ daily. Informed permission of the parents and the assent of the child were obtained.

The behavioral results were previously reported (Arnold et al. 1989). This report focuses on the blood lipid correlations with behavior.

Blood samples were collected before breakfast, at baseline and/or at the end of various treatment conditions, from 16 of the 18 original subjects. Blood draw was optional for extra payment each time; most subjects agreed every time; 1 declined at baseline but agreed later; some who agreed at baseline declined later; on a few occasions venipuncture was unsuccessful. The serum was separated and frozen at $-20^{\circ} \mathrm{C}$ for 2 years until analysis could be arranged at the laboratory of author R. A.G. Unfortunately, some of the specimens were lost.

Samples were extracted in 2:1 chloroform/methanol containing butylated hydroxyanisole $(0.005 \% \mathrm{w} / \mathrm{v})$ according to the method of Bligh and Dyer (1959). Lipid classes were separated from the total extracted lipids by thin-layer chromatography on silica-gel $\mathrm{H}$ plates developed in petroleum ether/diethyl ether/acetic acid (90:15:1 by volume). Bands containing total phospholipids, triglycerides, and cholesterol esters were scraped into vials containing $5 \mathrm{ml} \mathrm{1 \%}$ (v/v) sulfuric acid in methanol and the constituent fatty acids methylated by heating at $70^{\circ} \mathrm{C}$ for 3 hours. The resulting fatty-acid methyl esters were extracted into heptane and analyzed by capillary gas chromatography (Hewlett-Packard HP 5880 gas Chromatograph). The 50-meter glass column (0.56 mm I.D.) was coated with SP2340 (Supelco Inc., Bellefonte, PA) by Chromalytic Technology Limited (Boronia, Victoria, Australia). The esters were separated using a carrier helium flow of $30 \mathrm{~cm} / \mathrm{see}$ Avith a temperature gradient of $120^{\circ} \mathrm{C}$ to $200^{\circ} \mathrm{C}$ at $5^{\circ} \mathrm{C}$ per minute. The injection/split temperature was $250^{\circ} \mathrm{C}$ and the detector (F.I.D.) temperature was $300^{\circ} \mathrm{C}$. Fatty-acid methyl esters were identified against authentic lipid standards obtained from Nuchek Prep, Inc. (Elysian, Minnesota). Results were stated as percent of total fatty acids represented by linoleic acid, GLA, and DHGLA, respectively. This was done separately for serum triglycerides, phospholipids, and cholesterol esters.

Percentages of each fatty acid were correlated with concomitant behavior ratings. Spearman's correlations (SAS) were used to guard against skew by outliers.

To screen for a possible metabolic bottleneck at the rate-limiting enzyme (delta-6-desaturase), ratios were calculated of the amounts of the downstream metabolites to the amounts of the precursor linoleic acid. These ratios were then examined for correlation with the behavior ratings, in the same way as described with the raw percentages of each fatty acid. This was done for the ratio of metabolite GLA to precursor linoleic acid (GLA/linoleic) as well as for the ratio of the sum of GLA and dihomo-GLA to linoleic acid (GLA+ DHGLA/linoleic).

For some specimens the GLA level was below the detection threshold of $0.05 \%$. This problem, along with missing specimens (storage errors, random across conditions), reduced the $\mathrm{N}$ for many single-condition correlations to single digits. Therefore, attention was focused on correlations between lipid levels and behavior, analyzing across conditions and subjects. 


\section{Results}

Table 1 shows the correlations between four behavioral measures and three fatty-acid levels in the serum triglycerides. Note the significant negative correlations of behavior ratings with GLA but not its precursor linoleic acid in the serum triglycerides. Since higher behavior scores reflect more behavior problems, the negative behavioral correlation means that the higher serum GLA levels were associated with better behavior.

The levels of GLA correlated with the various behavioral ratings, with correlation coefficients (Spearman $r$ ) ranging from -0.47 to -0.59 (for each of the four behavioral ratings, $\mathrm{p}<$ $0.015)$. In contrast, the precursor linoleic acid correlated at levels which were generally nonsignificant ( $\mathrm{r}$ ranging from -0.12 to -0.28 ). This is the pattern predicted by the hypothesis. The correlations between behavioral ratings and the levels of dihomo-GLA were unremarkable.

\begin{tabular}{|c|c|c|c|c|c|c|c|c|c|c|}
\hline & \multicolumn{4}{|c|}{ Fatty acids } & \multirow{2}{*}{\multicolumn{2}{|c|}{$\begin{array}{c}\text { Dihomo-GLA } \\
\quad(20: 3 \Omega 6)\end{array}$}} & \multirow{2}{*}{\multicolumn{2}{|c|}{$\begin{array}{c}\text { Ratio of } \\
\text { GLA: linoleic }\end{array}$}} & \multirow{2}{*}{\multicolumn{2}{|c|}{$\begin{array}{c}\text { Ratio GLA + } \\
\text { dihomoGLA: } \\
\quad \text { linoleic }\end{array}$}} \\
\hline & \multirow{2}{*}{\multicolumn{2}{|c|}{$\begin{array}{l}\begin{array}{c}\text { Linoleic } \\
(18: 2 \Omega 6)\end{array} \\
N=59\end{array}$}} & \multirow{2}{*}{\multicolumn{2}{|c|}{$\begin{array}{c}\begin{array}{c}\text { Gamma-linolenic } \\
(18: 3 \Omega 26)\end{array} \\
N=27\end{array}$}} & & & & & & \\
\hline & & & & & $N$ & & & & & \\
\hline & $r^{b}$ & $p^{c}$ & $r$ & $p$ & $r$ & $p$ & $r$ & $p$ & $r$ & $p$ \\
\hline $\begin{array}{l}\text { Conners parent } \\
\text { questionnaire }\end{array}$ & -0.19 & 0.150 & -0.47 & 0.014 & -0.24 & 0.242 & -0.39 & 0.006 & -0.36 & 0.016 \\
\hline $\begin{array}{l}\text { Learning problem } \\
\text { factor }\end{array}$ & -0.28 & 0.034 & -0.59 & 0.001 & -0.36 & 0.075 & -0.35 & 0.013 & -0.23 & 0.131 \\
\hline $\begin{array}{l}\text { Hyperactivity } \\
\text { index }\end{array}$ & -0.12 & 0.358 & -0.50 & 0.009 & -0.29 & 0.148 & -0.423 & 0.002 & -0.37 & 0.012 \\
\hline $\begin{array}{l}\text { Davids hyper- } \\
\text { kinetic scale }\end{array}$ & -0.14 & 0.288 & -0.56 & 0.003 & -0.28 & 0.172 & -0.48 & 0.004 & -0.42 & 0.004 \\
\hline
\end{tabular}

Table 1. Correlation of Serum Triglyceride Fractions with Behavior

Correlations across conditions between specific serum triglyceride $£ 26$ acids and parent behavior ratings. A negative correlation means that better behavior is associated with higher serum level, $r=$ Spearman's rank order correlation; $p=$ probability (2-tailed); GLA = gamma-linolenic acid; $\mathrm{N}>26$ for each correlation. Both ratios (GLA/linoleic and the sum [GLA + Dihomo-GLA/linoleic) highlight the proportion of essential fatty-acid activity beyond the delta-6-desaturase metabolic bottleneck.

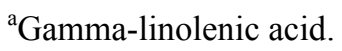

${ }^{\mathrm{b}}$ Spearman's rank-order correlation.

${ }^{c}$ Probability (2-tailed).

The ratios of the GLA and DHGLA metabolites to the precursor linoleic acid showed negative correlations to the behavior ratings; these correlations were generally significant (see Table 1).

Although the triglyceride GLA showed a significant negative correlation with behavior and learning problem indices, this pattern did not carry over in the phospholipids (see Table 2). There was a trend toward positive correlation of phospholipid GLA with behavioral scores which only reached statistical significance on the Parents Hyperactivity Index $(r=0.61, p<0.05)$. Similarly, the correlations of cholesterol GLA and behavior were unremarkable (see Table 3).

Clinical observations suggested that there were no significant or serious side effects in response to the treatment with GLA; two children had minor diarrhea, but it is unclear whether this 